\section{Production of actinium-225 \\ from a $(n, p)$ reaction: Feasibility and pre-design studies}

\author{
Fouad A. Abolaban (D), \\ Essam M. Banoqitah, \\ Eslam M. Taha, \\ Abdulsalam M. Alhawsawi, \\ Fathi A. Djouider, \\ Andrew Nisbet
}

\begin{abstract}
Actinium-225 is used in nuclear medicine for the treatment of malignant tumours. It can be applied to produce $\mathrm{Bi}-213$ in a reusable generator or can be used alone as an agent for radiation therapy, in particular for targeted alpha therapy. However, the availability of Ac-225 for worldwide use, particularly in low- and middle-income countries, is limited. We present a feasibility study employing GATE, an open-source Monte Carlo simulation toolkit, on the production of Ac-225 from a neutron generator. This work suggests that a design consisting of three concentric cylinders, the innermost a Cf-252 neutron source, the middle nickel cylinder acting as a proton-producing target and the outer cylinder a $\mathrm{RaCl}_{2}$ target may provide a feasible design outline for an Ac-225 generator.
\end{abstract}

Keywords: Actinium-225 • Radiopharmaceutical • Californium-252 • Neutron source • (n,p) reaction

\author{
F. A. Abolaban ${ }^{\bowtie}$, E. M. Banoqitah, F. A. Djouider \\ King Abdulaziz University \\ Faculty of Engineering \\ Department of Nuclear Engineering \\ P. O. Box 80204, Jeddah 21589, Saudi Arabia \\ E-mail: fabolaban@kau.edu.sa \\ E. M. Taha, A. M. Alhawsawi \\ King Abdulaziz University \\ Faculty of Engineering \\ Department of Nuclear Engineering \\ P. O. Box 80204, Jeddah 21589, Saudi Arabia \\ and King Abdulaziz University \\ Center for Training \& Radiation Prevention \\ P. O. Box 80204, Jeddah 21589, Saudi Arabia
}

\author{
A. Nisbet \\ University College London \\ Department of Medical Physics \& Biomedical \\ Engineering \\ Malet Place Engineering Building \\ London WC1E 6BT, UK
}

Received: 18 August 2020

Accepted: 6 April 2021

\section{Introduction}

Recent studies have shown that mortality among people due to prostate cancer is above 358000 worldwide, and is ranked as the second most occurrence of cancer after lung cancer [1-3]. Metastatic castration-resistant prostate cancer (mCRPC) is an advanced prostate cancer $[4,5]$. The prostate-specific membrane antigen (PSMA) is widely known as a glycoprotein cell surface that can be used as a metastatic prostate cancer indicator. Besides, the PSMA can be also used as a malignant neoplasms indicator of neovasculature [6]. This advanced stage of prostate cancer can be considered a non-curable disease depending on the PSMA spreading stage. Recent studies and clinical reports show that molecular radiotherapy using radionuclides such as $\mathrm{Lu}-177, \mathrm{Bi}-213$, and Ac-225 $[7,8]$ can increase the survival rate of patients $[7$, 9-12]. This involves labelling the PSMA with alpha or beta emitter sources where the alpha has a high linear energy transfer (LET) and relative biological effect (RBE) compare to beta. Actinium-225 is the preferred radionuclide as it is an alpha emitter and has both high LET and RBE almost 100 times more than beta emitters [6].

The generated amount of these radiopharmaceuticals is a major challenge as the demand is high while the supply is not sufficient [12]. Several studies were reported by Morgenstern et al. for producing Ac-225, most of which are still under research. Methods that have been proposed to pro-

0029-5922 @ 2021 The Author(s). Published by the Institute of Nuclear Chemistry and Technology.

This is an open access article under the CC BY-NC-ND 4.0 licence (http://creativecommons.org/licences/by-nc-nd/4.0/). 


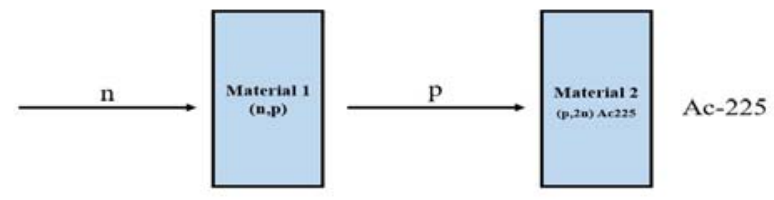

Fig. 1. Ac-225 generation process.

duce Ac-225 only serve as a ready-to-use Ac-225 in a vial or to have a generator to get Ac-225 which is known as an Ac-225/Bi-213 generator [10, 13, 14]. In addition, Bruchertseifer et al. showed the future demands and clinical applications for both Ac-225 and Bi-213 combined with PSMA for prostate cancer therapy [15]. Allen in 2017 published a comparative evaluation using Ac- 225 vs. Bi-213 as a therapeutic radioisotope where he reported that the Ac-225 has a superior therapeutic gain compared to $\mathrm{Bi}-213$ in terms of the amount of deposited energy [16]. The Ac-225 has a physical half-life of approximately ten days [17]. As a result, transporting this radionuclide to serve patient needs is difficult specifically in low and middle-income countries.

This paper aims to study the feasibility by first generating $\mathrm{p}$ in a thin layer of one material from an $(n, p)$ reaction, as shown in Fig. 1, and then Ac-225 atoms in a thin layer of a second material from a $(\mathrm{p}, 2 \mathrm{n})$ reaction. This study will evaluate the activation yield by utilizing different materials and the amount of Ac-225 gained from each material.

\section{Materials and methods}

Several research studies have been published explaining the different methods of generating Ac-225 [10, $14,18]$. The accelerator method that uses Ra-226 has been investigated theoretically and experimentally and shows promising results $[12,15,19]$. The target of $\mathrm{RaCl}_{2}$ can be irradiated by proton, neutrons or gamma rays [19]. In this work, the plan is to evaluate the generation of Ac-225 in a two-stage process using Ra-226 (p,2n) reaction: neutrons incident on material no. 1 shown previously in Fig. 1 to generate high energy protons, and the generated protons will then be incident on material no. 2, which is the $\mathrm{RaCl}_{2}$ in this study. The evaluation is carried out using the GATE Monte-Carlo simulation toolkit.

GATE incorporates the well-validated physics of GEANT4 with a more user-friendly simulation environment [20]. GATE has shown great potential in a wide range of medical applications, including radiotherapy [21-23], diagnostic radiology [24, 25], and dosimetry [26-28].

The simulation can be split into three stages. The first two stages focus on finding the optimum parameters for producing Ac-225 using a $\mathrm{RaCl}_{2}$ target (see Table 1), while the third employs these parameters into studying the feasibility and viability of producing the radionuclide using the said stages. The quark-gluon string model with a high neutron precision package QGSP_BIC_HP was used. This reference physics package provides a good description of secondary particles produced by protons and neutrons in addition to a very reliable transport modelling for neutrons below $20 \mathrm{MeV}$ [29].

\section{The first simulation stage}

The first simulation stage involves finding the proton energy threshold for producing Ac-225 by bombarding a $\mathrm{RaCl}_{2}$ target with high-energy protons. This is achieved by embedding a monoenergetic proton point source at the centre of a target composed of a radium chloride $\left(\mathrm{RaCl}_{2}\right)$ cylinder. Both the proton energy and the target thicknesses are varied to find the optimum conditions for producing Ac-225. Proton energies considered in this set of experiments are 2, 5, 7.5, 10, 15, and $20 \mathrm{MeV}$. Thicknesses considered are $0.04,0.06,0.08,0.1,0.25$, and $1 \mathrm{~mm}$. The number of particles used in this stage is $10^{8}$.

\section{The second simulation stage}

In the second simulation stage, the objective is to find the neutron energy and target material required to produce protons that meet the minimum requirements found in the previous step for producing Ac-225. Therefore, a neutron monoenergetic point

Table 1. Stages to find the optimum parameters for Ac-225 generation

\section{Simulation}

Stage 1: Bombarding $\mathrm{RaCl}_{2}$ target with high energy protons

Steps

1 2 3

Vary proton energy $\quad$ Vary $\mathrm{RaCl}_{2}$ target thickness Find optimum conditions to produce Ac-225

Stage 2: Bombarding several materials with neutrons to generate high energy protons found in the optimum condition for stage 1

Steps

1

Vary neutron energy

\section{2}

Vary material type and thickness
3

Find optimum conditions to generate high energy protons

Stage 3: Combining results from stages $1 \& 2$ to simulate two hollow cylinders: the inner cylinder is a proton-producing target, and the outer cylinder is $\mathrm{RaCl}_{2}$ to produce Ac-225

Steps

1

2

Proton production $(\mathrm{n}, \mathrm{p}) \quad$ Ac-225 production $(\mathrm{p}, 2 \mathrm{n})$ 
source is placed at the centre of a cylindrical proton-producing target. The neutron energy, cylinder material and its thickness are varied. For each run, the energy spectrum of the produced protons is recorded. The feasibility of each neutron energy, material type and thickness depends on the quantity and energy of the produced protons. Several materials were investigated, including nickel $(\mathrm{Ni})$, iron $(\mathrm{Fe})$, potassium (K), stainless-steel (SS), magnesium (Mg) and manganese $(\mathrm{Mn})$. The number of particles used in this stage was $10^{8}$.

\section{The final simulation stage}

The outcomes of the first and second stages form the basis of the third stage. The setup of the third stage consists of two hollow co-axial cylinders. The inner cylinder acts as the proton-producing target. The proton-producing target composition and thickness are dependent on the outcomes of the second stage. The outer cylinder represents the target and is composed of $\mathrm{RaCl}_{2}$ with the optimum thickness found in the first stage. At the centre of the proton-producing target, a point neutron source is placed. All available neutron sources with energies meeting the criteria are used against this setup to find the probability of producing Ac-225 for each neutron source.

The number of neutrons used should be high enough to produce enough protons to produce Ac-225. The fraction of protons produced by neutrons and Ac-225 produced by protons is expected to be relatively low. Therefore, a very high number of neutrons is required to produce Ac-225. Using a very high number of particles would extend the simulation time significantly. Alternatively, to achieve low statistical uncertainty in calculating Ac- 225 production probability while keeping the simulation time reasonable, the simulation is split into two parts.

The splitting is carried out by adding a very thin virtual layer $(1 \mu \mathrm{m})$ between the two cylinders. The purpose of this layer is to record the properties of all protons produced in the proton-producing target and leaving its volume towards the target.

When the proton-producing target is bombarded with $10^{8}$ neutrons, some protons will be produced. Several of these protons will leave the proton-producing target and transverse the thin layer where their momentum and spatial properties are stored in a phase space file. Subsequently, the simulation is relaunched using the same setup but with the neutron source being replaced by a proton source. This proton source emits from the phase-space layer with the source properties derived from the phase-space file. The number of protons emitted from the phase-space file is set to $5 \times 10^{6}$ and by that, the number of protons is amplified by a factor ranging approximately from $10^{4}$ to $10^{5}$.

\section{Results and discussion}

The cross section for Ac-225 production from $\mathrm{RaCl}_{2}$ material depends mainly on the proton energy inter-

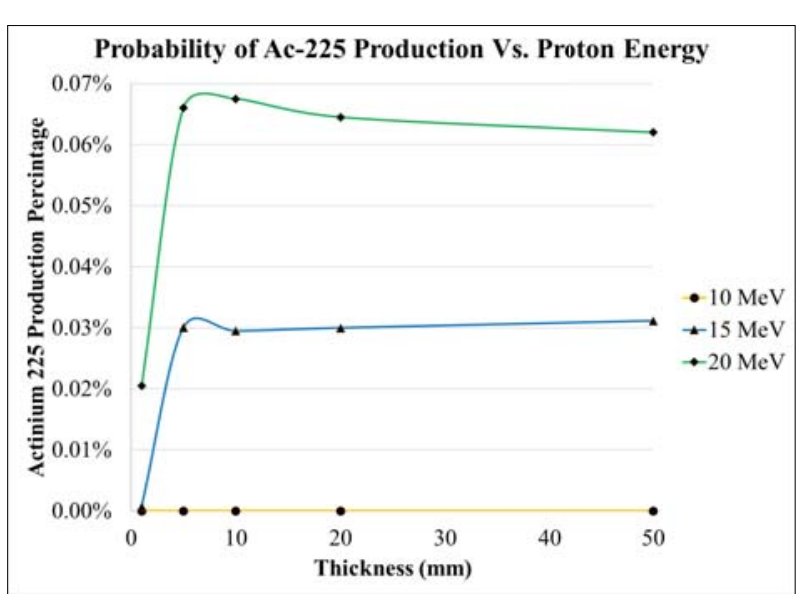

Fig. 2. Probability of producing Ac-225 from bombarding $\mathrm{RaCl}_{2}$ with high energy protons inside the cylinder target as a function of proton energy. The target density is $4.9 \mathrm{~g} / \mathrm{cm}^{3}$. An isotropic point proton source was placed inside a $\mathrm{RaCl}_{2}$ cylinder with a $3 \mathrm{~cm}$ height and varying diameter (thickness) in an air volume.

acting with $\mathrm{RaCl}_{2}$. Figure 2 shows the dependence of producing Ac- 225 on the proton energy. Even though six proton energies were simulated in the first stage, starting at $2.5 \mathrm{MeV}$, the figure only shows three curves because the probability of producing Ac-225 from protons with energies below or equal to $10 \mathrm{MeV}$ is between $0.0006 \%$ and $0.0008 \%$. The optimum $\mathrm{RaCl}_{2}$ thickness to produce Ac-225 for $15 \mathrm{MeV}$ and $20 \mathrm{MeV}$ protons was determined to be $5 \mathrm{~mm}$. To calculate the activity of the generated Ac-225 using the curves from Fig. 2, a 1 Ci proton source incident on a $5 \mathrm{~mm}$ thick $\mathrm{RaCl}_{2}$ target will result in $32 \mu \mathrm{Ci}$ and $68 \mu \mathrm{Ci}$ for the $10 \mathrm{MeV}$ and the $15 \mathrm{MeV}$ proton energies, respectively.

The generation of high-energy protons is crucial in producing actinium as shown in the previous figure. To produce high-energy protons inside the Ac-225 generator, a neutron beam with energies from $10 \mathrm{MeV}$ up to $20 \mathrm{MeV}$ was incident on nickel $(\mathrm{Ni})$, iron $(\mathrm{Fe})$, potassium $(\mathrm{K})$, stainless-steel (SS), magnesium (Mg) and manganese (Mn). The following discussion is for nickel, manganese and iron as they produced the best results.

Figure 3 shows the proton average energy generated at different nickel thicknesses from a neutron beam with $10,12.5,15,17.5$, and $20 \mathrm{MeV}$. It should

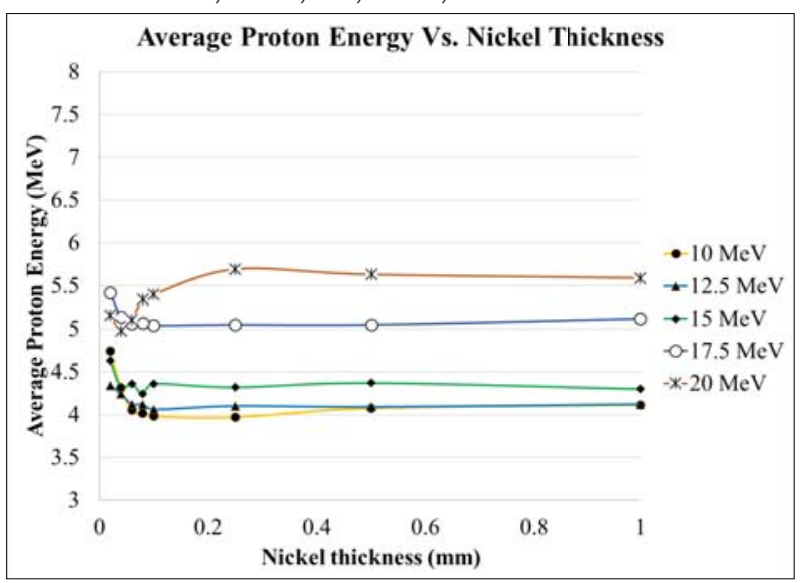

Fig. 3. Average proton energy generated from neutron beams interacting with nickel at several thicknesses. 


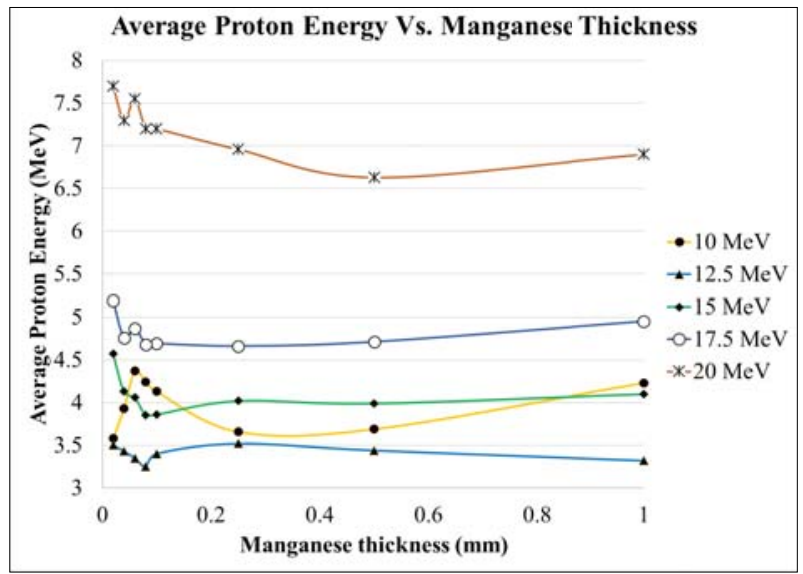

Fig. 4. Average proton energy generated from neutron beams interacting with manganese at several thicknesses.

be noted that the maximum proton energy generated cannot exceed the maximum neutron energy from the $(\mathrm{n}, \mathrm{p})$ reaction. The figure demonstrates that the higher the neutron energy, the higher the average proton energy generated at all suggested nickel thickness. The maximum thickness in the $x$ axis for Fig. 4 is $1 \mathrm{~mm}$ because thicker materials would absorb the proton since charged particles start to interact as soon as they are generated.

The average produced protons energy ranges from 0 to approximately the maximum incident neutron energy, i.e. a $10 \mathrm{MeV}$ neutron can produce $10 \mathrm{MeV}$ protons, because of the conservation of energy. The incident neutron energy and the average produced proton energy are directly proportional. The maximum average proton energy was around $5.6 \mathrm{MeV}$ at $0.25 \mathrm{~mm}$ from the $20 \mathrm{MeV}$ neutrons.

Figure 4 shows the average proton energy generated at different manganese thicknesses from a neutron beam of 10, 12.5, 15, 17.5, and $20 \mathrm{MeV}$. Figure 4 confirms what was demonstrated in the nickel case, where the higher the neutron energy, the higher the average proton energy generated is at all thicknesses. The maximum thickness was kept to $1 \mathrm{~mm}$ to reduce the probability of proton absorption in the manganese.

The maximum average proton energy generated by $20 \mathrm{MeV}$ neutrons is approximately $7.7 \mathrm{MeV}$ at $\sim 0.05 \mathrm{~mm}$, which is higher than the nickel case.

Figure 5 shows the average proton energy generated at different iron thicknesses from a neutron beam of $10,12.5,15,17.5$, and $20 \mathrm{MeV}$. Figure 5 follows the same trend observed in the nickel and manganese cases, where the higher the incident neutron energy is, the higher the average proton energy is generated at all thicknesses. The maximum average proton energy generated by $20 \mathrm{MeV}$ neutrons is around $6 \mathrm{MeV}$ at $\sim 0.05 \mathrm{~mm}$, which is slightly higher than the nickel case.

Figure 6 shows the probability of producing protons at the same nickel thicknesses used in Fig. 3 as a function of neutron energy. The probability is maximum at $0.1 \mathrm{~mm}$ thickness for the $17.5 \mathrm{MeV}$ neutron energy and slowly decreases as the nickel thickness increases for all other neutron energies.

Figure 7 shows the probability of producing protons at different manganese thicknesses as a func-

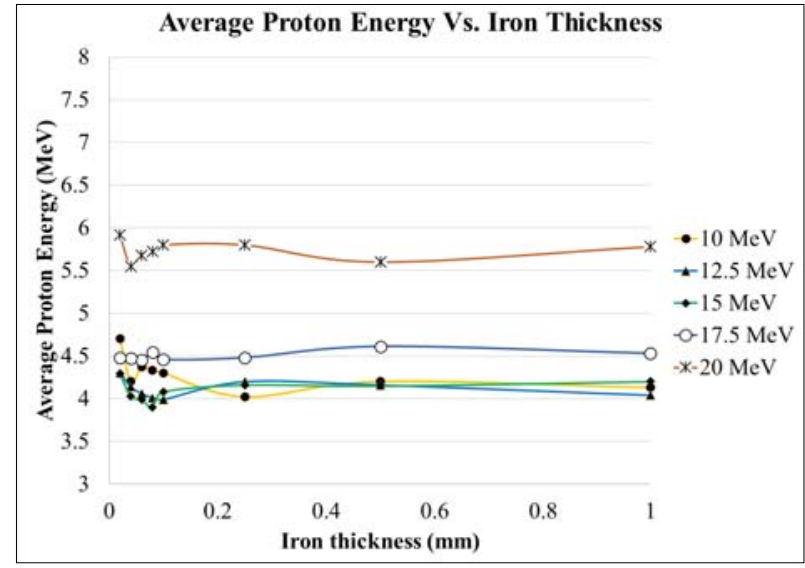

Fig. 5. Average proton energy generated from neutron beams interacting with iron at several thicknesses.

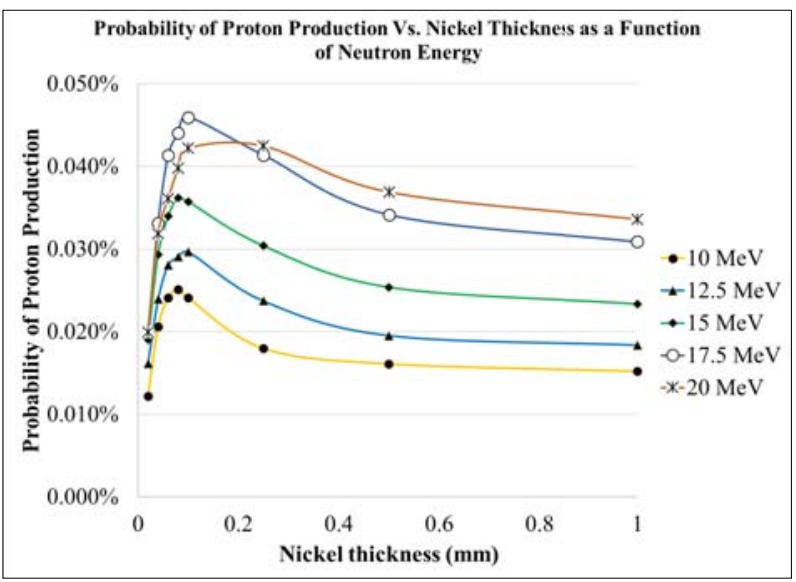

Fig. 6. Probability of proton production from neutrons incident on nickel at different thicknesses as a function of neutron energy. The target density is $8.9 \mathrm{~g} / \mathrm{cm}^{3}$. An isotropic point neutron source was placed inside a $\mathrm{RaCl}_{2}$ cylinder with a $3 \mathrm{~cm}$ height and varying diameter (thickness) in an air volume.

tion of neutron energy. The probability is maximum at $0.25 \mathrm{~mm}$ thickness for the $20 \mathrm{MeV}$ neutron energy and decreases as the neutron energy decreases.

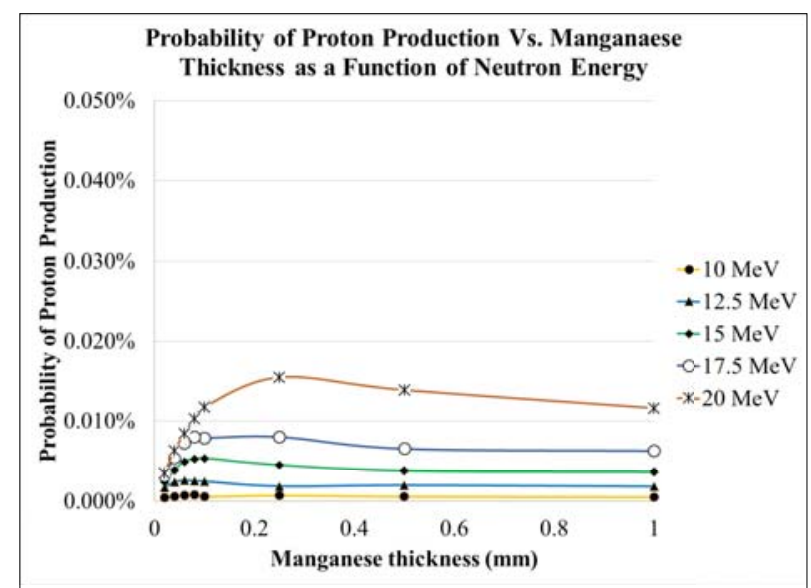

Fig. 7. Probability of proton production from neutrons incident on manganese at different thicknesses as a function of neutron energy. The target density is $7.43 \mathrm{~g} / \mathrm{cm}^{3}$. An isotropic point neutron source was placed inside a $\mathrm{RaCl}_{2}$ cylinder with a $3 \mathrm{~cm}$ height and varying diameter (thickness) in an air volume. 


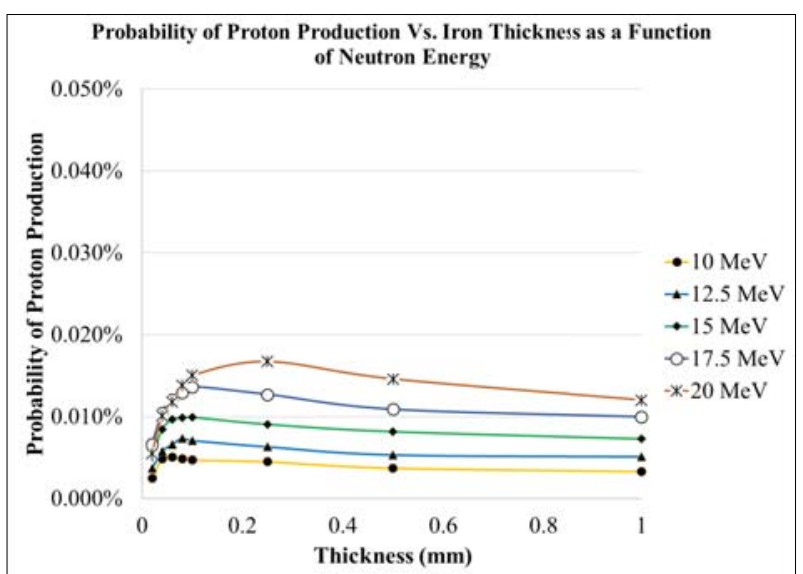

Fig. 8. Probability of proton production from neutrons incident on iron at different thicknesses as a function of neutron energy. The target density is $7.87 \mathrm{~g} / \mathrm{cm}^{3}$. An isotropic point neutron source was placed inside an $\mathrm{RaCl}_{2}$ cylinder with a $3 \mathrm{~cm}$ height and varying diameter (thickness) in an air volume.

There is a tradeoff between the maximum average proton energy that can be produced by nickel and manganese and the probability of producing the protons for the two metals.

Figure 8 shows the probability of producing protons at different iron thicknesses as a function of neutron energy. The probability is maximum at $0.25 \mathrm{~mm}$ thickness for the $20 \mathrm{MeV}$ neutron energy and decreases as the neutron energy decreases. The iron and manganese have similar proton production probabilities, both are equal but less than the nickel case.

The results for potassium, stainless-steel, and magnesium are not discussed here because either the probability of proton production is very low so that it is not feasible to bombard the $\mathrm{RaCl}_{2}$ with sufficient protons to generate Ac-225, or the generated proton energy is not enough to produce Ac-225. Combining the results for nickel, manganese and iron, it can be concluded that the neutron source must possess energy $>10 \mathrm{MeV}$ to produce Ac-225 in addition to a high probability of producing protons. Thus, nickel was chosen as the target for the neutron source inside the generator. The steps needed to generate Ac-225 are shown in Fig. 9. The first two steps of the process are made from three concentric cylinders. The innermost is a Cf-252 source, the middle cylinder is made from nickel and the outermost cylinder is made from $\mathrm{RaCl}_{2}$. A chemical separation stage is needed to be applied for each batch produced by the generator that contains Ac-225 to get rid of the unwanted fragments generated by the irradiation process in the third step before preparing a patient dose in the fourth and final step. Additional shielding should be placed around the generator to protect the users from neutrons and gamma rays.

Since Cf-252 emits a spectrum of energies, additional simulations were run to find the optimum nickel thickness between $0.01 \mathrm{~mm}$ and $0.06 \mathrm{~mm}$. The $0.01 \mathrm{~mm}$ thickness produced $3.3 \mathrm{MeV}$ average proton energy and the $0.05 \mathrm{~mm}$ thickness produced 2.7 $\mathrm{MeV}$ average energy with about 90\% more protons than the $0.01 \mathrm{~mm}$ thickness. Thus, the $0.05 \mathrm{~mm}$ thickness of nickel was chosen to be paired with Cf-252. On the other hand, all $\mathrm{RaCl}_{2}$ thicknesses produced a similar amount of Ac-225, and so a $5 \mathrm{~mm}$ thickness was used. Even though it was stated at the beginning of the results section that the proton energy needed to produce Ac-225 should be $>10 \mathrm{MeV}$, this does not contradict the $2.7-3.3 \mathrm{MeV}$ average proton energy generated from bombarding nickel with Cf- 252 because the maximum generated proton energy from the simulation was between $10 \mathrm{MeV}$ and $19 \mathrm{MeV}$ for the $0.05 \mathrm{~mm}$ nickel thickness depending on the neutron energy.

The simulation results demonstrated that in order to produce a single Ac-225 atom: (1) a Cf-252 source with a minimum activity of $7.15 \mathrm{E} 12 \mathrm{~Bq}$ should be used, (2) the neutrons from the Cf-252 should be incident on a nickel target with a thickness of $0.05 \mathrm{~mm}$, (3) the nickel target will produce a $2.7 \mathrm{MeV}$ average energy protons, (4) the protons will be incident on an $\mathrm{RaCl}_{2} 5 \mathrm{~mm}$ thick target. A further set of simulations were carried out replacing the Cf-252 by a typical neutron generator with a $14 \mathrm{MeV}$ energy. The geometry for the generator simulation differs from the Cf-252 one. The generator will be incident on a $5 \mathrm{~mm}$ height and $5 \mathrm{~mm}$ diameter cylinder needle with a $0.05 \mathrm{~mm}$ nickel thickness. The nickel needle material in the needle will produce high-energy protons that are incident on $5 \mathrm{~mm} \mathrm{RaCl}$ volume inside the cylinder shape needle as this was experimentally tested [30]. The Ac-225 atoms are produced at the back of the $\mathrm{RaCl}_{2}$ volume. The minimum activity needed to produce a single Ac-225 atom/s with the neutron generator is $2.21 \mathrm{E} 9 \mathrm{~Bq}$.

The Cf-252 and the neutron generator produced isotopes/fragments in addition to the Ac-225. Some of the isotopes are radioactive and some are not, so the chemical separation stage is of great importance to produce a pure Ac-225 solution that does not contain other radioactive materials to ensure the dose delivered to the patient is optimized.

In terms of real-life applicability, the process of producing Ac-225 is feasible. Because of the high activity Cf-252 source, there will be a need
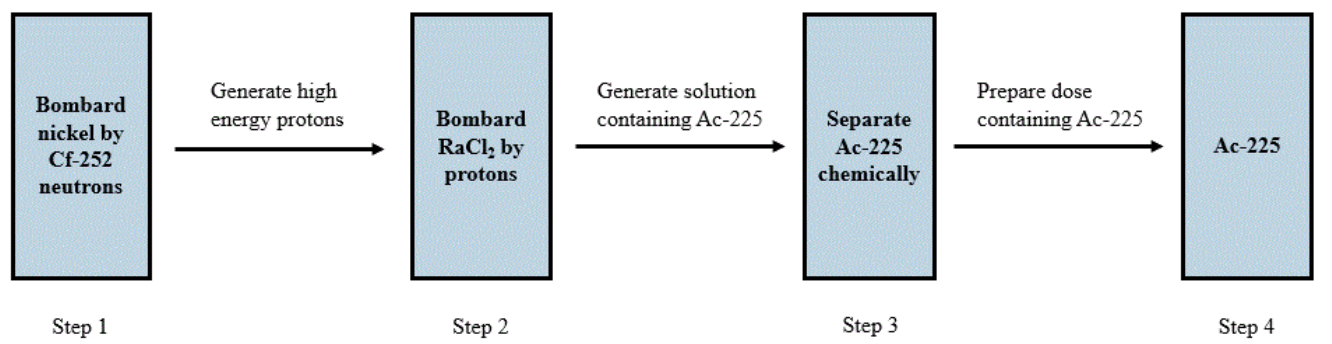

Fig. 9. Proposed Ac-225 generator. 
to shield against the high energy neutrons and the high energy gamma emitted by the source. A two-metre diameter barrel shield made of high-density polyethylene (HDPE) with a small lead cylinder around the Cf-252 source will be used to shield against the neutrons and gamma radiations. A solution containing Ac-225 will be generated from the apparatus containing the neutron source; nickel and $\mathrm{RaCl}_{2}$ cylinders will generate batches that will require chemical separation. Once Ac-225 is separated, the radiation dose will be prepared to use for patients. Further studies are needed to determine the amount of $\mathrm{Ra}$ in the $\mathrm{RaCl}_{2}$ solution/target. An automated system will be required to optimize the user radiation exposure to unnecessary radiation dose [31].

Two of the most important fragments/isotopes generated in the solution containing Ac-225 are Ac-224 $\left(T_{1 / 2}=2.9 \mathrm{~h}\right)$ and Ac-226 $\left(T_{1 / 2}=29 \mathrm{~h}\right)$. Those isotopes have very short half-lives compared to the Ac-225 and they depend on the energy of the proton bombarded into the $\mathrm{RaCl}_{2}$.

\section{Conclusion}

It has been demonstrated by employing GATE, an open-source Monte Carlo simulation toolkit, that the production of Ac-225 from a neutron generator is feasible. This work suggests that a design consisting of three concentric cylinders, the innermost a Cf-252 neutron source, the middle nickel cylinder acting as a proton-producing target and the outer cylinder a $\mathrm{RaCl}_{2}$ target may provide a feasible design outline for an Ac-225 generator. The optimum dimensions for the proton-producing target are $0.05 \mathrm{~mm}$ nickel and the optimum $\mathrm{RaCl}_{2}$ thickness to produce Ac-225 was determined to be $5 \mathrm{~mm}$. Such a design concept may help address the issue of a lack of radiopharmaceutical production to address worldwide demand, particularly in low and middle-income countries.

Acknowledgments. This project was funded by the Deanship of Scientific Research (DSR) at King Abdulaziz University, Jeddah, under grant no. RG-28-135-38. The authors, therefore, gratefully acknowledge DSR's technical and financial support.

\section{ORCID}

F. A. Abolaban (D) http://orcid.org/0000-0002-7434-1663

\section{References}

1. Villers, A., \& Grosclaude, P. (2008). Épidémiologie du cancer de la prostate. Med. Nucl., 32(1), 2-4. DOI:10.1016/j.mednuc.2007.11.003.

2. Global Cancer Observatory. (2019). Prostate 2018. Available from https://gco.iarc.fr/today/data/factsheets/cancers/27-Prostate-fact-sheet.pdf.

3. Bray, F., Ferlay, J., Soerjomataram, I., Siegel, R. L., Torre, L. A., \& Jemal, A. (2018). Global cancer sta- tistics 2018: GLOBOCAN estimates of incidence and mortality worldwide for 36 cancers in 185 countries. CA Cancer J. Clin., 68(6), 394-424. DOI:10.3322/ caac. 21492.

4. Davison, B. J., Gleave, M. E., Goldenberg, S. L., Degner, L. F., Hoffart, D., \& Berkowitz, J. (2002). Assessing information and decision preferences of men with prostate cancer and their partners. Cancer Nurs., 25(1), 42-49.

5. Hofman, M. S., Violet, J., Hicks, R. J., Ferdinandus, J., Thang, S. P., Akhurst, T., Iravani, A., Kong, G., Kumar, A. R., Murphy, D. G., Eu, P., Jackson, P., Scalzo, M., Williams, S. G., \& Sandhu, S. (2018). [177 $\mathrm{Lu}$ ]-PSMA-617 radionuclide treatment in patients with metastatic castration-resistant prostate cancer (LuPSMA trial): a single-centre, single-arm, phase 2 study. Lancet Oncol., 19(6), 825-833. DOI:10.1016/ S1470-2045(18)30198-0.

6. Li, Y., Tian, Z., Rizvi, S., Bander, N., Allen, B., \& Wales, S. (2002). In vitro and preclinical targeted alpha therapy of human prostate cancer with Bi-213 labeled J591 antibody against the prostate specific membrane antigen. Prostate Cancer Prostatic Dis., 5, 36-46. DOI:10.1038/sj/pcan/4500543.

7. Azorín-Vega, E., Rojas-Calderón, E., Ferro-Flores, G., Aranda-Lara, L., Jiménez-Mancilla, N., \& NavaCabrera, M. A. (2019). Assessment of the radiation absorbed dose produced by 177 Lu-iPSMA, 225 AciPSMA and $223 \mathrm{RaCl} 2$ to prostate cancer cell nuclei in a bone microenvironment model. Appl. Radiat. Isot., 146, 66-71. DOI:10.1016/j.apradiso.2019.01.020.

8. Ahn, J., Park, S., Zuniga, B., Bera, A., Song, C. S., \& Chatterjee, B. (2016). Vitamin D in prostate cancer. In Vitamins and hormones (Vol. 100, pp. 321-355). New York: Academic Press Inc.

9. Ludwig, D. L., Bryan, R. A., Dawicki, W., Geoghegan, E. M., Liang, Q., Gokhale, M., Reddy, V., Garg, R., Allen, K. J. H., Berger, M. S., \& Dadachova, E. (2020). Preclinical development of an actinium-225-labeled antibody radio-conjugate directed against CD45 for targeted conditioning and radioimmunotherapy. Biol. Blood Marrow Transplant., 26(3), S160-S161. DOI:10.1016/j.bbmt.2019.12.714.

10. Miederer, M., Scheinberg, D. A., \& McDevitt, M. R. (2008). Realizing the potential of the actinium-225 radionuclide generator in targeted alpha particle therapy applications. Adv. Drug Deliv. Rev., 60(12), 1371-1382.

11. Dekempeneer, Y., Keyaerts, M., Krasniqi, A., Puttemans, J., Muyldermans, S., Lahoutte, T., D'huyvetter, M., \& Devoogdt, N. (2016). Targeted alpha therapy using short-lived alpha-particles and the promise of nanobodies as targeting vehicle. Expert Opin. Biol. Ther., 16(8), 1035-1047. DOI:10.1080/14712598.2 016.1185412 .

12. Morgenstern, A., Apostolidis, C., \& Bruchertseifer, F. (2020). Supply and clinical application of actinium-225 and bismuth-213. Semin. Nucl. Med., 50(2), 119-123. DOI:10.1053/j.semnuclmed.2020.02.003.

13. Artun, O. (2017). Estimation of the production of medical Ac-225 on thorium material via proton accelerator. Appl. Radiat. Isot., 127, 166-172. DOI:10.1016/j.apradiso.2017.06.006.

14. Kossert, K., Takács, M. P., \& Nähle, O. (2020). Determination of the activity of ${ }^{225} \mathrm{Ac}$ and of the half-lives 
of ${ }^{213} \mathrm{Po}$ and ${ }^{225} \mathrm{Ac}$. Appl. Radiat. Isot., 156, 109020. DOI:10.1016/j.apradiso.2019.109020.

15. Bruchertseifer, F., Kellerbauer, A., Malmbeck, R., \& Morgenstern, A. (2019). Targeted alpha therapy with bismuth-213 and actinium-225: Meeting future demand. J. Label. Compd. Radiopharm., 62(11), 794-802. DOI:10.1002/jlcr.3792.

16. Allen, B. J. (2017). A comparative evaluation of Ac225 vs Bi213 as therapeutic radioisotopes for targeted alpha therapy for cancer. Australas. Phys. Eng. Sci. Med., 40(2), 369-376. DOI:10.1007/s13246-017-0534-6.

17. Ruddy, F. H., Dulloo, A. R., Seidel, J. G., \& Petrović, B. (2004). Separation of the alpha-emitting radioisotopes actinium-225 and bismuth-213 from thorium-229 using alpha recoil methods. Nucl. Instrum. Methods Phys. Res. Sect. B: Beam Interact. Mater. Atoms, 213, 351-356. DOI:10.1016/S0168583X(03)01580-5.

18. Artun, O. (2017). Estimation of the production of medical Ac-225 on thorium material via proton accelerator. Appl. Radiat. Isot., 127, 166-172. DOI:10.1016/j.apradiso.2017.06.006.

19. Apostolidis, C., Molinet, R., McGinley, J., Abbas, K., Möllenbeck, J., \& Morgenstern, A. (2005). Cyclotron production of Ac-225 for targeted alpha therapy. Appl. Radiat. Isot., 62(3), 383-387. DOI:10.1016/j. apradiso.2004.06.013.

20. Jan, S., Santin, G., Strul, D., Staelens, S., Assie, K., Autret, D., Avner, S., Barbier, R., Bardies, M., Bloomfield, P. M., Brasse, D., Breton, V., Bruyndonckx, P., Buvat, I., Chatziioannou, A. F., Choi, Y., Chung, Y. H., Comtat, C., Donnarieix, D., Ferrer, L., Glick, S. J., Groiselle, C. J., Guez, D., Honore, P. F., KerhoasCavata, S., Kirov, A. S., Kohli, V., Koole, M., Krieguer, M., van der Laan, D. J., Lamare, F., Largeron, G., Lartizien, C., Lazaro, D., Maas, M. C., Maigne, L., Mayet, L., Melot, F., Merheb, C., Pennacchio, E., Perez, J., Pietrzyk, U., Rannou, F. R., Rey, M., Schaart, D. R., Schmidtlein, C. R., Simon, L., Song, T. Y., Vieira, J. M., Visvikis, D., Van der Walle, R., Wieers, E., \& Morel, C. (2004). GATE: a simulation toolkit for PET and SPECT. Phys. Med. Biol., 49(19), 4543-4561. DOI:10.1088/0031-9155/49/19/007.

21. Grevillot, L., Bertrand, D., Dessy, F., Freud, N., \& Sarrut, D. (2011). A Monte Carlo pencil beam scanning model for proton treatment plan simulation using GATE/GEANT4. Phys. Med. Biol., 56(16), 5203-5219. DOI:10.1088/0031-9155/56/16/008.

22. Grevillot, L., Boersma, D. J., Fuchs, H., Aitkenhead, A., Elia, A., Bolsa, M., Winterhalter, C., Vidal, M.,
Jan, S., Pietrzyk, U., Maigne, L., \& Sarrut, D. (2020). Technical Note: GATE-RTion: a GATE/Geant4 release for clinical applications in scanned ion beam therapy. Med. Phys., 47(8), 3675-3681. DOI:10.1002/ mp.14242.

23. Aitelcadi, Z., Toufique, Y., El Kharrim, A., Elmadani, S., Hilali, A., \& Bouhali, O. (2018). Validation of the GATE Monte Carlo code for radiation therapy: Varian Clinac2300C/D. In Proceedings of the 2018 International Conference on Optimization and Applications, ICOA 2018, 31 May 2018, pp. 1-4. Institute of Electrical and Electronics Engineers Inc. DOI:10.1109/ ICOA.2018.8370602.

24. Aguiar, P., Casarejos, E., Silva-Rodriguez, J., Vilan, J. A., \& Iglesias, A. (2015). Geant4-GATE simulation of a large plastic scintillator for muon radiography. IEEE Trans. Nucl. Sci., 62(3), 1233-1238. DOI:10.1109/ TNS.2015.2431297.

25. Lamare, F., Turzo, A., Bizais, Y., Le Rest, C. C., \& Visvikis, D. (2006). Validation of a Monte Carlo simulation of the Philips Allegro/GEMINI PET systems using GATE. Phys. Med. Biol., 51 (4), 943-962. DOI:10.1088/0031-9155/51/4/013.

26. Papadimitroulas, P. (2017). Dosimetry applications in GATE Monte Carlo toolkit. Phys. Medica, 41, 136-140. DOI:10.1016/j.ejmp.2017.02.005.

27. Thiam, C. O., Breton, V., Donnarieix, D., Habib, B., \& Maigne, L. (2008). Validation of a dose deposited by low-energy photons using GATE/GEANT4. Phys. Med. Biol., 53(11), 3039-3055. DOI:10.1088/00319155/53/11/019.

28. Villoing, D., Marcatili, S., Garcia, M. -P., \& Bardiès, M. (2017). Internal dosimetry with the Monte Carlo code GATE: validation using the ICRP/ ICRU female reference computational model. Phys. Med. Biol., 62(5), 1885-1904. DOI:10.1088/13616560/62/5/1885.

29. Geant4 Collaboration. (2018). Physics reference manual. CERN

30. Maslov, O. D., Sabel'nikov, A. V., \& Dmitriev, S. N. (2006). Preparation of ${ }^{225} \mathrm{Ac}$ by ${ }^{226} \mathrm{Ra}(\gamma, \mathrm{n})$ photonuclear reaction on an electron accelerator, MT-25 microtron. Radiochemistry, 48(2), 195-197. DOI:10.1134/ S1066362206020184.

31. Balasundar, S., Chandrasekaran, S., Subramanian, V., \& Venkatraman, B. (2021). Investigations on neutron attenuation properties of poly-boron materials using Am-Be and ${ }^{252} \mathrm{Cf}$ sources neutron spectra. Ann. Nucl. Energy, 153, 108083. DOI:10.1016/j. anucene.2020.108083. 